\title{
Conditional PTEN-deficient Mice as a Prostate Cancer Chemoprevention Model
}

\author{
Hiroyuki Koike ${ }^{1}$, Masahiro Nozawa ${ }^{1 *}$, Marco A De Velasco ${ }^{1,2}$, Yurie Kura ${ }^{1}$, \\ Naomi Ando ${ }^{1}$,Emiko Fukushima ${ }^{1}$, Yutaka Yamamoto ${ }^{1}$, Yuji Hatanaka ${ }^{1}$, Kazuhiro \\ Yoshikawa $^{3}$, Kazuto Nishio ${ }^{2}$, Hirotsugu Uemura ${ }^{1}$
}

\begin{abstract}
Background: We generated a mouse model of prostate cancer based on the adult-prostate-specific inactivation of phosphatase and tensin homolog (PTEN) using the Cre-loxP system. The potential of our mice as a useful animal model was examined by evaluating the chemopreventive efficacy of the anti-androgen, chlormadinone acetate (CMA). Materials and Methods: Six-week-old mice were treated subcutaneously with $50 \mu \mathrm{g} / \mathrm{g}$ of CMA three times a week for 9 or 14 weeks and sacrificed at weeks 15 and 20. Macroscopic change of the entire genitourinary tract (GUT) and histologically evident prostate gland tumor development were evaluated. Proliferation and apoptosis status in the prostate were examined by immunohistochemistry. Results: CMA triggered significant shrinkage of not only the GUT but also prostate glands at 15 weeks compared to the control $(p=0.017$ and $p=0.010$, respectively), and the trend became more marked after a further five-weeks of treatment. The onset of prostate adenocarcinoma was not prevented but the proliferation of cancer cells was inhibited by CMA, which suggested the androgen axis is critical for cancer growth in these mice. Conclusions: Conditional PTEN-deficient mice are useful as a preclinical model for chemoprevention studies and serve as a valuable tool for the future screening of potential chemopreventive agents.
\end{abstract}

Keywords: Animal model - chlormadinone acetate - prevention - prostate cancer - PTEN

Asian Pac J Cancer Prev, 16 (5), 1827-1831

\section{Introduction}

Prostate cancer $(\mathrm{PCa})$ is the most common cancer in men in the United States (Siegel et al., 2014). An estimated 233,000 new cases will be diagnosed in 2014, accounting for $27 \%$ of new cancer cases in men in 2014. An increasing trend of PCa has also been the case with Asian countries (Daniyal et al., 2014). PCa incidence more than doubled from 2.2 per 100,000 men in 2000 to 4.7 per 100,000 men in 2010 in Vietnam (Dong et al., 2014). Age-adjusted PCa incidence rate was 35 cases per 100,000 in 2009 in Turkey (Zorlu et al., 2014). Androgen deprivation therapy (ADT) consisting of a gonadotropin-releasing hormone agonist or antagonist or surgical castration with or without an anti-androgen is commonly effective as primary systemic therapy in advanced disease, however, the disease may eventually become resistant to ADT in most patients, that is the development of castration-resistant PCa (CRPC) (Pienta et al., 2006).

Treatment duration of CRPC recently tends to become extended with prolonged patients' survival due to newlydeveloped drugs such as abiraterone acetate, enzalutamide, and cabazitaxel (de Bono et al., 2010; Fizazi et al., 2012; Scher et al., 2012). These new drugs are generally expensive and $\mathrm{PCa}$ has been a significant burden on healthcare resources in worldwide. From the standpoint of both cancer control and medical economy, the prophylaxis of PCa can be an ideal solution to the situation.

Recently, several reports suggested that metabolic syndrome and hyperlipidemia were associated with PCa occurrence (McGrowder et al., 2012; Tewari et al., 2014), and diets with high fat and sugar are associated to the increasing risk of PCa (Askari et al., 2014a). Intake of fruits, vegetables, and especially isoflavone may reduce the risk of PCa occurrence (Sugiyama et al., 2013; Zhou et al., 2013; Askari et al., 2014b). Various studies had showed positive, negative or null effect on reducing PCa risk with the use of vitamins $\mathrm{A}, \mathrm{D}$ and $\mathrm{E}$ and selenium (Yang et al., 2013; Garg et al., 2014). Even randomized trials and intervention studies vary in their results in determining the role of vitamins in PCa. Thus, these types of studies need a large number of patients, funds, and time to prove a PCa-preventive efficacy of a candidate agent. Therefore, a fruitful strategy would entail the development of a faithful animal model that more accurately represents the human disease in order to investigate the potential chemopreventive compounds of PCa.

We generated a mouse model of PCa based on the 
prostate-specific inactivation of phosphatase and tensin homolog (PTEN) using the Cre-loxP system (De Velasco et al., 2014). In this model, mice develop prostate cancer in a stage-specific manner that includes all stages of the disease process. Furthermore latency is short with half of the mice developing cancers at 10 weeks of age and all mice developing adenocarcinomas by 20 weeks of age. Here, we examined the potency of our mice as a $\mathrm{PCa}$ animal model by evaluating the chemopreventive efficacy of chlormadinone acetate (CMA), a steroidal anti-androgen similar to progesterone that inhibits the uptake of testosterone by epithelial cells and the binding of dihydrotestosterone (DHT) to androgen receptors (ARs) (Kodama et al., 1980).

\section{Materials and Methods}

\section{Reagents and antibodies}

CMA is kindly provided from ASKA Pharmaceutical Co.ltd. (Tokyo, Japan). Antibodies for immunohistochemistry (IHC), PCNA (\#RB-9055) and AR (\#RB-9030), were purchased from Thermo Scientific (Waltham, MA, USA).

\section{Animals}

Prostate-specific PTEN-deficient mice (C57/B) were used for this experiments. This animal model has been developed and organized in our laboratory. Briefly, using the Cre-loxP system we generated PSA-driven PTEN homozygous-deletion mice that exhibit a series of tumor development and progression in the dorsolateral lobes of the prostatic gland. These mice develop prostatic intraepithelial neoplasia (PIN) as early as 8 weeks of age and a half of these mice progress to develop adenocarcinomas microscopically by 10 weeks. All mice develop cancer by 20 weeks and steady progression of disease result in distant metastasis in a half of mice by 75 weeks. The characterization of this mouse system has been described elsewhere (De Velasco et al., 2014). All animal experiments using this model were approved by the institutional review committee at Kinki University Faculty of Medicine.

\section{Experimental design}

To assess the effects of CMA on development and progression of PCa in our PTEN-deficient mouse model, we carried out a chemoprevention experiment with CMA. Six-week-old mice were treated subcutaneously 3 times a week with $50 \mu \mathrm{g} / \mathrm{g}$ of CMA for 9 or 14 weeks. Mice were sacrificed at week 15 and 20 ( $n=7$, respectively) (Figure 1).

\section{Tumor burden and efficacy evaluation}

Drug antitumor effects were determined by comparing differences in genitourinary tract (GUT) weight or prostate surface area as described previously (De Velasco et al., 2014). Briefly, the GUT was harvested en bloc and weighed fresh. The prostate surface area was determined by 2 -D morphometric analysis using digital images of GUTs captured by a Nikon Coolpix 995 camera connected to an Olympus SX61 stereomicroscope. Area measurements were calculated on spatially calibrated images using Image-J image analysis software (http:// imagej.nih.gov/ij/).

\section{Pathological assessment and Immunohistochemistry}

GUTs were fixed in formalin and embedded in paraffin. Paraffin sections were stained with hematoxylin/ eosin (HE) for pathological diagnosis and examined immunohistochemically for AR and PCNA. Pathological classification of murine prostate cancer was carried out according to the criteria of the Bar Harbor Classification system (Shappell et al., 2004; Ittmann et al., 2013). Tumor distribution analysis was analyzed using photomicrographs of GUT cross sections (HE, 40X). Briefly, the digital images were stitched with Photoshop CS5 Extended (Adobe System, Sab Jose, CA, USA) and the masks corresponding to stroma, normal acini, PIN and adenocarcinoma in the dorsolateral and ventral lobes of the prostate were generated. The area of each mask was recorded and calculated. Immunohistochemical evaluation was done by comparing $\%$ positive staining rates. Assessment of staining was carried out using Image-J analysis software.

\section{Statistical analysis}

Statistical analyses were carried out by Student t-test for pairwise comparison and one way ANOVA for multiple comparisons using SigmaStat 3.5 (Systat Software, San Jose, CA, USA). Differences were determined significant if the p-value was less than 0.05 .

\section{Results}

Morphological changes of the genitourinary tracts and tumor development glands by chlormadinone acetate

Figure 2A shows the macroscopic views of GUT from 15-week-old mice of the CMA treatment group and the control. The GUTs of CMA-treated mice were markedly diminished and their weights were significantly smaller than those of the control group at 15 and 20 weeks after birth $(\mathrm{p}=0.017$ and $\mathrm{p}=0.009$, respectively; Figure $2 \mathrm{~B})$. CMA treatment caused the significant loss of GUT weight from 15 to 20 weeks old ( $p=0.025$; Figure $2 B$ ) contrary to the growing trend in the control group. The prostate glands also shrank significantly in the CMAtreated group compared to the control mice, based on the 2-D morphometric analysis, at 15 and 20 weeks of age $(p=0.010$ and $p<0.001$ respectively; Figure $2 C)$. The prostate area decreased in the CMA-treated mice from 15 to 20 weeks old in contrast to the control group $(\mathrm{p}=0.005$; Figure 2C).

\section{Experimental Design}

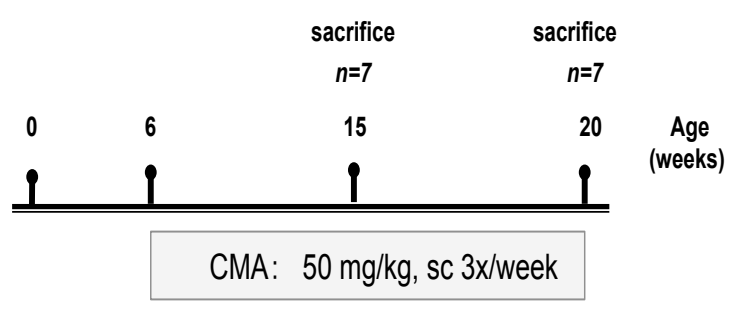

Figure 1. Experimental Design. CMA was Administered

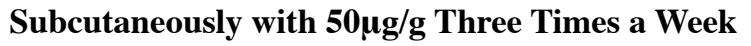



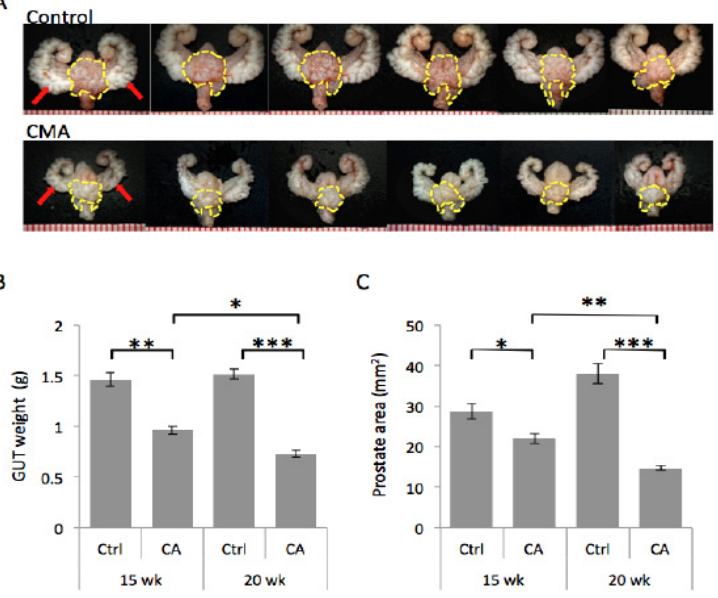

Figure 2. A) Macroscopic Views of the Whole Genitourinary Tracts (GUTs). Areas surrounded by the yellow dotted line indicate the dorsolateral and ventral lobes of the prostate. Seminal vesicles are indicated by red arrows, scale is in mm. B) Comparison of the GUT weights. * $\mathrm{p}=0.025$, $* * \mathrm{p}=0.017, * * * \mathrm{p}=0.009$. C) Comparison of the prostate gland areas. $* \mathrm{p}=0.010, * * \mathrm{p}=0.005, * * * \mathrm{p}<0.001$
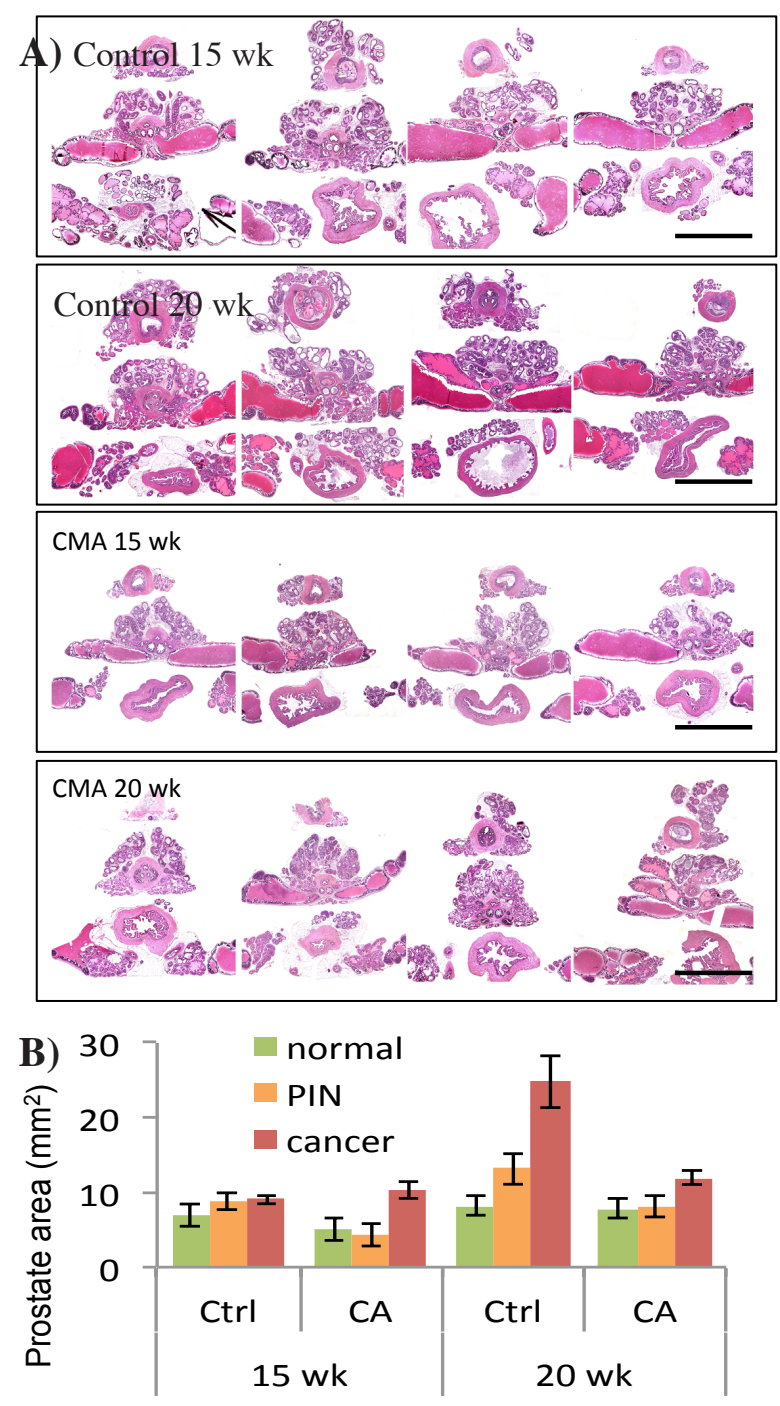

Figure 3. A) Hematoxylin/eosin (HE) Staining of the GUTs. The dorsolateral and ventral lobes of the prostate are shown with other adjacent organs. Scale bars represent $5 \mathrm{~mm}$. B) Tissue distribution in the prostate glands
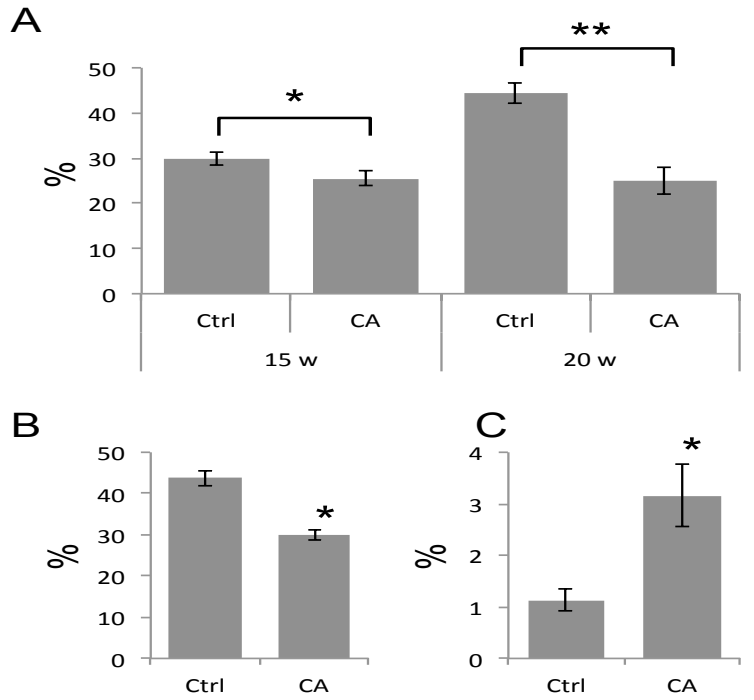

Figure 4. Quantitative Analysis for Immunohistochemistry in the Prostate Glands. A) Transition of AR into the nuclei. ${ }^{*} \mathrm{p}=0.044, * * \mathrm{p}<0.001$. B) PCNA expression. ${ }^{*} \mathrm{p}<0.001$. C) TUNEL assay. ${ }^{*} \mathrm{p}=0.004$

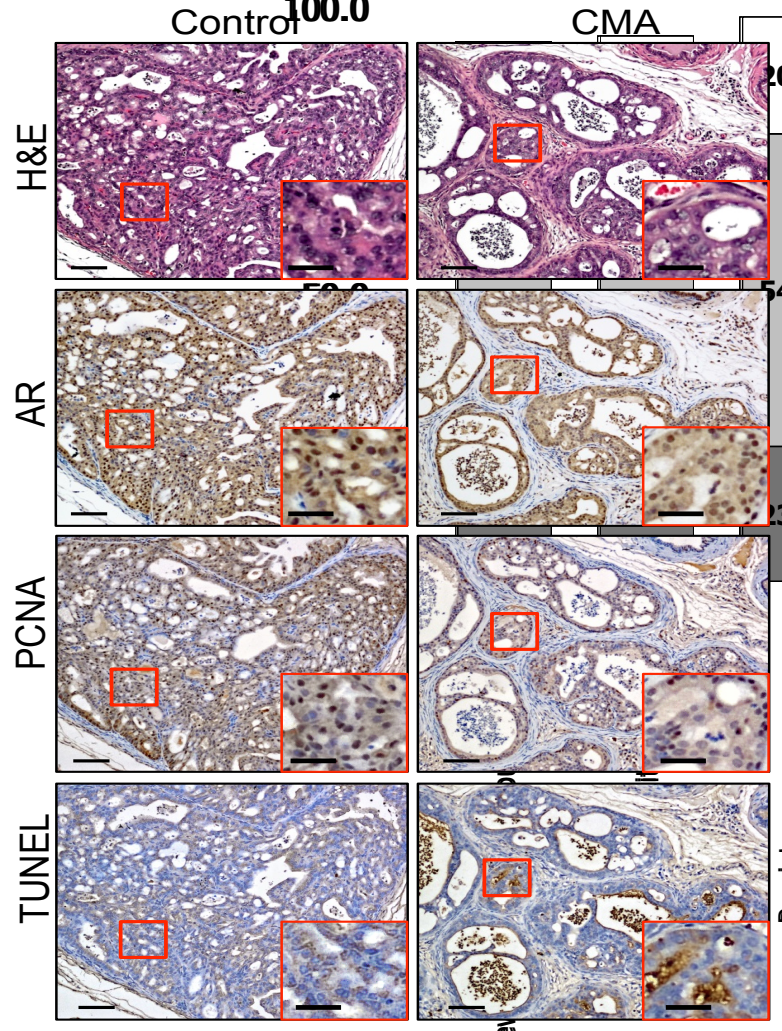

Figure 5. HE Staining and Immunohistochemical Analyses on AR, PCNA, and TUNEL in the Prostate Glands. Areas in the squares of red line are magnified. Scale bars represent $200 \mu \mathrm{m}$ in low magnification and $100 \mu \mathrm{m}$ in inserts

\section{Histological effects of chlormadinone acetate}

Figure 3A shows the HE staining of the whole GUTs and prostate glands from 15 and 20 week-old mice of CMA and control groups. In the control group, normal prostate glands, PIN and adenocarcinoma lesions appeared almost equally at 15 weeks, and both PIN and adenocarcinoma markedly increased from 15 to 20 weeks. PIN and adenocarcinoma were also detected at 15 weeks in the 
CMA group. The ratio of PIN rather increased, whereas that of adenocarcinoma did not, from 15 to 20 weeks in the CMA group. These findings were confirmed by the computer-processed analysis for tissue distribution in the serial sections of the prostate glands (Figure 3B).

Inhibition of proliferation and induction of apoptosis to the prostate glands by chlormadinone acetate

The nuclear transition of ARs, confirmed by IHC, was significantly lowered in the CMA group than the control group at 15 weeks $(\mathrm{p}=0.044)$ and the trend was more overt at 20 weeks $(\mathrm{p}<0.001$; Figures $4 \mathrm{~A}$ and 5$)$. Furthermore, the proliferation rates based on the expression of PCNA in prostatic adenocarcinoma cells was suppressed in the CMA group compared with the control group at 20 weeks of age $(\mathrm{p}<0.001$ : Figures $4 \mathrm{~B}$ and 5). Apoptotic rates in adenocarcinomas, measured by the TUNEL assay, were induced in the CMA group compared to the control group at 20 weeks of age $(\mathrm{p}=0.004$; Figures $4 \mathrm{C}$ and 5).

\section{Discussion}

CMA blocks the AR axis by inhibiting the uptake of testosterone in prostatic epithelial cells and the binding of DHT to ARs. Based on this therapeutic mechanism, CMA has been historically used in the systemic treatment for advanced PCa mainly in the combination with medical or surgical castration as well as benign prostatic hyperplasia to diminish the prostate volume (Matsumoto et al., 2014). Our present study reasonably showed that the decrease of GUT weight and the shrinkage of prostatic tissue in the mice treated with CMA compared with the control group (Figure 2). The percentage of cancer lesions in the whole prostate gland was also reduced in the treatment group (Figure 3). These findings likely resulted from the blockade of AR signal by CMA, based on the inhibition of AR transition into the nuclei leading to the proliferative suppression and the apoptotic induction in PCa cells (Figures 4 and 5). However, CMA treatment suppressed the proliferation of cancer cells but did not decrease the development of PIN, which means CMA did not work to prevent $\mathrm{PCa}$ onset. The findings from present study suggest that inhibiting the androgen signal is effective to prevent proliferation of $\mathrm{PCa}$ raised by PTEN dysfunction, and that androgen plays an important role from the early stage of PCa development in this mouse model. There is abundant evidence that androgens influence the development of PCa also in human beings. Therefore the present findings suggest that prostate-specific $P T E N$-deficient mice we generated are useful as a PCadeveloping animal model not only morphologically but also in terms of pathogenesis.

The present study was performed using geneticallymanipulated PTEN-deficient mice. PTEN is broadly expressed during development and adulthood and encodes a lipid phosphatase that functions as an inhibitor of the phosphoinositide 3-kinase (PI3K)/AKT pathway (Maehama et al., 1998). PTEN function lost as a result of mutations, deletions, or promoter methylation silencing, occurs at a high frequency in human prostate cancer and correlates with increased AKT-1 phosphorylation; and is consequently associated with a poor prognosis and initiates downstream targets that modulate a wide range of cellular processes associated with the progression of tumor cell growth and survival (Yoshimoto et al., 2007; McCall et al., 2008; Davies et al., 2011). The advantage of our $P T E N$-deficient mice is the use of human PSA promoter for expression of Cre that causes PTEN inactivation only in the luminal cells of the adult prostate (De Velasco et al., 2014). Contrary to probasin-based promoters that target both basal and luminal cells of prostate glands and the seminal vesicles in developing and mature animals, the normal development of prostate glands during immature generation is not disturbed in our model mice (Parisotto et al., 2013). Our mice have a favorable property as a prostate cancer model in terms of both organ- and timespecific PTEN deletion. PCa prevention experiments using Transgenic Adenocarcinoma of Mouse Prostate (TRAMP) mice have been reported, however, tumors from these mice show a histological phenotype type that more closely resembles neuroendocrine tumors (Saw et al., 2011).

In summary, we have used a clinically relevant mouse model of prostate cancer to illustrate the chemopreventive effects of targeting the AR axis. Furthermore we provide proof of concept for the use of this model as a screening tool for potential chemopreventive agents. Thus, the prostate-specific PTEN-deficient mouse model represents useful tool for the preclinical evaluation and characterization of promising chemopreventive agents.

\section{Acknowledgements}

This work was supported by Institutional Funds. The authors thank all members in the laboratory of Department of Urology for their help in experiments.

\section{References}

Askari F, Parizi MK, Jessri M, et al (2014a). Dietary patterns in relation to prostate cancer in Iranian men: a case-control study. Asian Pac J Cancer Prev, 15, 2159-63.

Askari F, Parizi MK, Jessri M, et al (2014b). Fruit and vegetable intake in relation to prostate cancer in Iranian men: a casecontrol study. Asian Pac J Cancer Prev, 13, 5223-7.

Daniyal M, Siddiqui ZA, Akram M, et al (2014). Epidemiology, etiology, diagnosis and treatment of prostate cancer. Asian Pac J Cancer Prev, 15, 9575-8.

Davies MA (2011). Regulation, role, and targeting of Akt in cancer. J Clin Oncol, 29, 4715-7.

De Bono JS, Oudard S, Ozguroglu M, et al (2010). Prednisone plus cabazitaxel or mitoxantrone for metastatic castrationresistant prostate cancer progressing after docetaxel treatment: a randomised open-label trial. Lancet, 376, 1147-54.

De Velasco MA, Tanaka M, Yamamoto Y, et al (2014). Androgen deprivation induces phenotypic plasticity and promotes resistance to molecular targeted therapy in a PTEN-deficient mouse model of prostate cancer. Carcinogenesis, 35, 214253.

Dong HV, Lee AH, Nga NH, et al (2014). Epidemiology and prevention of prostate cancer in Vietnam. Asian Pac J Cancer Prev, 15, 9747-51.

Fizazi K, Scher HI, Molina A, et al (2012). Abiraterone acetate for treatment of metastatic castration-resistant prostate 
cancer: final overall survival analysis of the COU-AA-301 randomised, double-blind, placebo-controlled phase 3 study. Lancet Oncol, 13, 983-92.

Garg M, Dalela D, Goel A, et al (2014). Prevention of prostate cancer with vitamins-current perspectives. Asian Pac J Cancer Prev, 15, 1897-904.

Ittmann M, Huang J, Radaelli E, et al (2013). Animal models of human prostate cancer: the consensus report of the New York meeting of the mouse models of human cancers consortium prostate pathology committee. Cancer Res, 73, 2718-36.

Kodama T, Wakisaka M, Shimazaki J (1980). Binding of chlormadinone acetate to cytosol from human benign prostatic hypertrophy. Endocrinol Jpn, 27, 53-7.

Maehama T, Dixon JE (1998). The tumor suppressor, PTEN/ MMAC1, dephosphorylates the lipid second messenger, phosphatidylinositol 3,4,5-trisphosphate. J Biol Chem, 273, 13375-8.

McCall P, Witton CJ, Grimsley S, et al (2008). Is PTEN loss associated with clinical outcome measures in human prostate cancer? Br J Cancer, 99, 1296-301.

McGrowder DA, Jackson LA, Crawford TV (2012). Prostate cancer and metabolic syndrome: is there a link? Asian Pac J Cancer Prev, 13, 1-13.

Parisotto M, Metzger D (2013). Genetically engineered mouse models of prostate cancer. Mol Oncol, 7, 190-205.

Pienta KJ, Bradley D (2006). Mechanisms underlying the development of androgen-independent prostate cancer. Clin Cancer Res, 12, 1665-71.

Saw CL, Wu TY, Paredes-Gonzalez X, et al (2011). Pharmacodynamics of fish oil: protective effects against prostate cancer in TRAMP mice fed with a high fat western diet. Asian Pac J Cancer Prev, 12, 3331-4.

Scher HI, Fizazi K, Saad F, et al (2012). Increased survival with enzalutamide in prostate cancer after chemotherapy. $N$ Engl J Med, 367, 1187-97.

Shappell SB, Thomas GV, Roberts RL, et al (2004). Prostate pathology of genetically engineered mice: definitions and classification. the consensus report from the bar harbor meeting of the mouse models of human cancer consortium prostate pathology committee. Cancer Res, 64, 2270-305.

Siegel R, Ma J, Zou Z, et al (2014). Cancer statistics, 2014. CA Cancer J Clin, 64, 9-29.

Sugiyama Y, Masumori N, Fukuta F, et al (2013). Influence of isoflavone intake and equol-producing intestinal flora on prostate cancer risk. Asian Pac J Cancer Prev, 14, 1-4.

Tewari R, Chhabra M, Natu SM, et al (2014). Significant association of metabolic indices, lipid profile, and androgen levels with prostate cancer. Asian Pac J Cancer Prev, 15, 9841-6.

Yang L, Pascal M, Wu XH (2013). Review of selenium and prostate cancer prevention. Asian Pac J Cancer Prev, 14, 2181-4.

Yoshimoto M, Cunha IW, Coudry RA, et al (2007). FISH analysis of 107 prostate cancers shows that PTEN genomic deletion is associated with poor clinical outcome. Br J Cancer, $\mathbf{9 7}$, 678-85.

Zhou XF, Ding ZS, Liu NB (2013). Allium vegetables and risk of prostate cancer: evidence from 132,192 subjects. Asian Pac J Cancer Prev, 14, 4131-4. 